\title{
ARTICLE
}

Received 3 Oct 2014 | Accepted 10 Apr 2015 | Published 15 May $2015 \quad$ DOl: 10.1038/ncomms8156

\section{Coherent control of plasma dynamics}

\author{
Z.-H. He ${ }^{1}$, B. Hou' ${ }^{1}$ V. Lebailly², J.A. Nees ${ }^{1}$, K. Krushelnick ${ }^{1} \&$ A.G.R. Thomas ${ }^{1}$
}

Coherent control of a system involves steering an interaction to a final coherent state by controlling the phase of an applied field. Plasmas support coherent wave structures that can be generated by intense laser fields. Here, we demonstrate the coherent control of plasma dynamics in a laser wakefield electron acceleration experiment. A genetic algorithm is implemented using a deformable mirror with the electron beam signal as feedback, which allows a heuristic search for the optimal wavefront under laser-plasma conditions that is not known a priori. We are able to improve both the electron beam charge and angular distribution by an order of magnitude. These improvements do not simply correlate with having the 'best' focal spot, as the highest quality vacuum focal spot produces a greatly inferior electron beam, but instead correspond to the particular laser phase front that steers the plasma wave to a final state with optimal accelerating fields.

\footnotetext{
${ }^{1}$ Center for Ultrafast Optical Science, University of Michigan, Ann Arbor, Michigan 48109-2099, USA. 2 Polytech Paris-Sud-Université Paris-Sud, 91405

Orsay, France. Correspondence and requests for materials should be addressed to Z.-H.H. (email: zhhe@umich.edu).
} 
T he concept of coherent control-precise measurement or determination of a process through control of the phase of an applied oscillating field-has been applied to many different systems, including quantum dynamics ${ }^{1}$, trapped atomic ions $^{2}$, chemical reactions ${ }^{3}$, Cooper pairs ${ }^{4}$, quantum $\operatorname{dots}^{5,6}$ and $\mathrm{THz}$ generation ${ }^{7}$ to name but a few. A plasma wave is a coherent and deterministically evolving structure that can be generated by the interaction of laser light with plasma. It is therefore natural to assume that coherent control techniques may also be applied to plasma waves. Plasma waves produced by high-power lasers have been studied intensively for their numerous applications, such as the production of ultrashort pulses by plasma wave compression $^{8,9}$, generation of extremely high-power pulses by Raman amplification ${ }^{10,11}$, for inertial confinement fusion ignition schemes $^{12,13}$, as well as for fundamental scientific investigations. In particular, laser wakefield acceleration of ultra-relativistic electron beams ${ }^{14-17}$, has been a successful method for accelerating electrons to relativistic energies over a very short distance. In laser wakefield acceleration, an electron bunch 'surfs' on the electron plasma wave generated by an intense laser and gains a large amount of energy. The accelerating electric field strength that the plasma wave can support can be many orders of magnitude higher than that of a conventional accelerator, which makes laser wakefield acceleration an exciting prospect as an advanced accelerator concept. However, although highly competitive in terms of accelerating gradient, beams from laser wakefield accelerator experiments are currently inferior to conventional accelerators in terms of other important characteristics, such as energy spread and stability. In addition, owing to constraints in laser wakefield technology, experimental demonstrations have predominantly been performed in single shot operation, far below the $\mathrm{kHz}-\mathrm{MHz}$ repetition rates of conventional accelerators.

In recent years, deformable mirror adaptive optical systems have been successfully implemented in high-intensity laser experiments to increase the peak laser intensity by improving the beam focusability, especially in systems using high numerical aperture optics. The shape of the deformable mirror is generally determined in a closed loop where either a direct measurement of the wavefront is performed ${ }^{18}$ or some nonlinear optical signal $^{19,20}$ is used as feedback in an iterative algorithm. The objective of adaptive optics has largely been optimization of the laser focal shape to a near diffraction-limited spot, thus producing

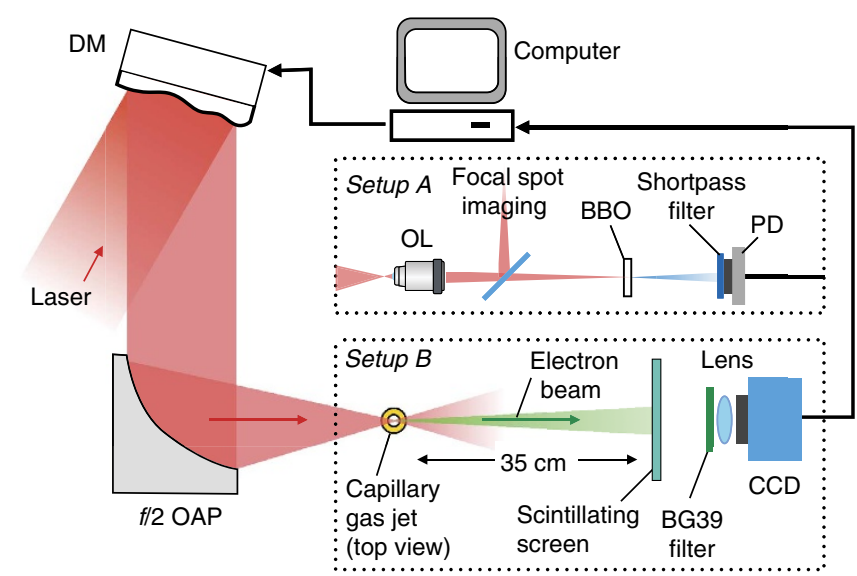

Figure 1 | Experimental setup. DM, deformable mirror; OAP, off-axis parabolic mirror, $50 \mathrm{~mm}$ diameter; OL, objective lens; PD, photodiode. A: Conventional focal spot optimization using second-harmonic generation; B: setup for direct optimization of the electron signal from the laser plasma accelerator. the highest possible intensity. Adaptive optics can also be useful for certain focal profile shaping ${ }^{21,22}$, optimization of a laser machining process ${ }^{23}$ or harmonic generation ${ }^{24,25}$.

In the following, we demonstrate that orders of magnitude improvement to electron beam properties from a laser wakefield accelerator operating at $\mathrm{kHz}$ repetition rate can be made, through the use of a genetic algorithm coupled to a deformable mirror adaptive optical system to coherently control the plasma wave formation. The electron image from a scintillator screen was processed and used in the fitness function as feedback for the genetic algorithm. Using this method, we were able to improve the beam properties significantly. This result was not simply due to an improvement in focal quality since a laser pulse with the 'best' (highest intensity/lowest $M^{2}$ ) focus in vacuum produced a greatly inferior electron beam compared with a laser pulse optimized using the electron beam properties themselves. It was found that the focal spot optimized for electron beam production had pronounced intensity 'wings'. Modifications to the phase front of the tightly focusing laser alter the light propagation, which experiences strong optical nonlinearities in the plasma, and therefore affect the plasma wave dynamics in a complex but deterministic manner.

\section{Results}

Experimental setup and procedure. The experiment was performed using the relativistic Lambda-Cubed $\left(\lambda^{3}\right)$ laser system (see Methods). The output laser beam was reflected from a deformable mirror and focused onto a free-flowing argon gas plume to produce an electron beam by laser wakefield acceleration (see Methods) at $500 \mathrm{~Hz}$. Electrons were measured using a scintillating screen imaged onto a lens-coupled CCD camera. The experimental setup is shown schematically in Fig. 1.

We first implemented the genetic algorithm for laser focus optimization using the second-harmonic signal generated from a beta barium borate crystal (setup A in Fig. 1). The laser spot was optimized such that highest peak intensity is achieved when the second harmonic generation is strongest. Subsequently, we modified the fitness function to use a figure of merit (FOM, refer to equation 1 in Methods) from the electron scintillation data, calculating the inverse distance weighting (with power parameter $n$ ) to a single point $\mathbf{r}_{0}$ for all pixel intensities within an electron image. The pixel of the optimization point $\mathbf{r}_{0}$ was dynamically adjusted during the genetic algorithm to concentrate all electron signal to the peak location of the charge distribution during each generation. The genetic algorithm was initialized using a 'flat' mirror shape with $30 \mathrm{~V}$ for all actuators to allow immediate deformation in both directions.

Optimization of the electron spatial profile. For comparison, electron beams produced by the 'best' laser focus (by optimizing the intensity) and the initial mirror shape at $30 \mathrm{~V}$ are shown in Fig. 2a,b, respectively. The optimized electron beam profiles are shown in Fig. $2 \mathrm{c}-\mathrm{h}$ for various weighting parameters, $n$. The genetic algorithm converged to the best electron beam using $n=8$ in terms of beam divergence and peak charge density. The peak charge density was increased by a factor of 20 compared with the initial electron beam profile before optimization (see Fig. 2j). The optimized electron profile is highly stable and collimated, with a full-width-at-half-maximum (FWHM) divergence of $\Delta x=7.4 \pm 0.6 \mathrm{mrad}$ and $\Delta y=12.8 \pm 1.4 \mathrm{mrad}$. The shot-to-shot pointing (defined by the centroid position) fluctuation of the electron beam is less than $1 \mathrm{mrad}$ (root mean square). The integrated charge was increased by more than twofold from the electron beams generated by a laser focus of highest intensity. The high repetition rate and real-time diagnostics permit 

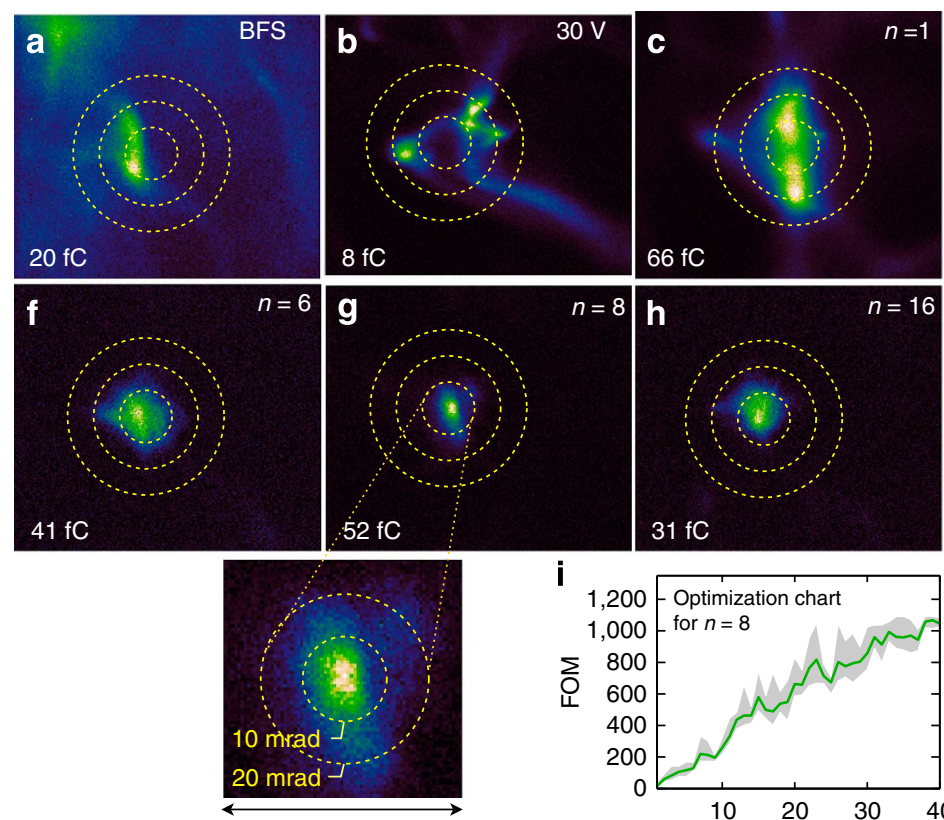

$10 \mathrm{~mm}$

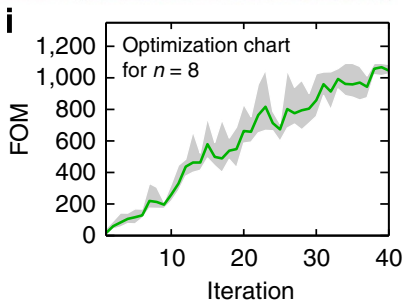

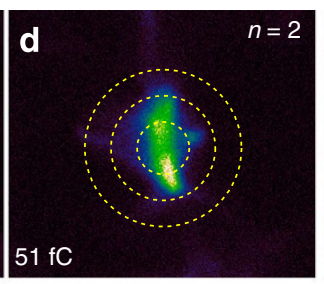

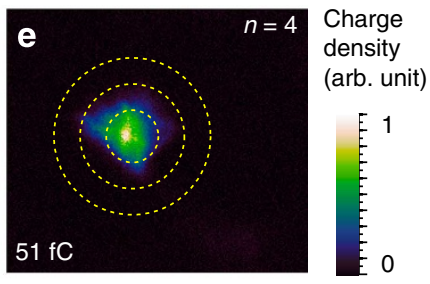

j

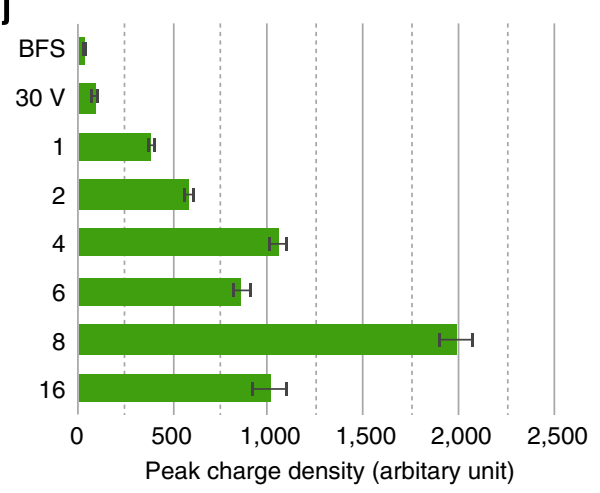

Figure 2 | Optimization of the electron spatial profile. Electron beam profile image integrated over 50 shots (100 ms exposure time) with a deformable mirror configuration (a) corrected for the best focal spot (BFS) and (b) $30 \mathrm{~V}$ on all actuators. (c-h) Single-shot electron beam profiles after genetic algorithm optimization using different weighting parameters, $n$. (i) The convergence of the genetic algorithm with $n=8$. The shaded grey area represents the range of the ten best children in each iteration and the solid green curve is the average. (j) Comparison of the peak charge density in a single-shot electron image. Error bars represent the root mean square shot-to-shot fluctuations; estimated charge per shot is displayed for every image. Contours shown are for 20,40, 60 mrad, centred on the beam centroid.

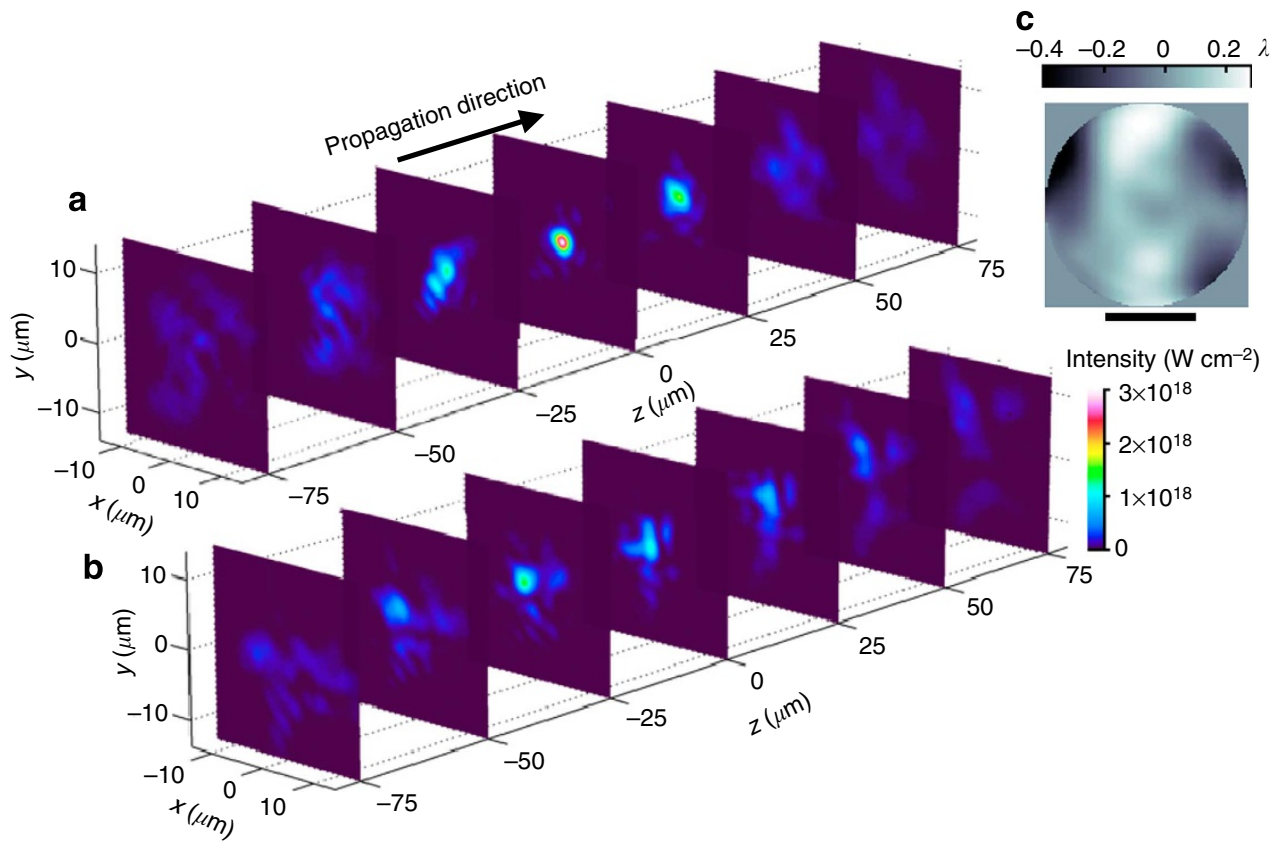

Figure 3 | Laser intensity profile in the focal region and wavefront. Scan of laser focal intensity in vacuum with the deformable mirror optimized for (a) second harmonic signal (highest intensity) and (b) electron beam in Fig. 2. (c) Relative wavefront change reconstructed from direct measurement using a Shack-Hartmann wavefront sensor. Root-mean-square phase deviation in the aperture is 0.14 wave. The wavefront was reconstructed over a slighly smaller aperture $(2.26 \mathrm{~mm}$ diameter $)$ than the full beam diameter on the sensor $\left(2.7 \mathrm{~mm} 1 / \mathrm{e}^{2}\right.$ width $)$ to reduce errors in the peripheral area (scale bar, $1 \mathrm{~mm}$ ).

implementation of the algorithm within a practical time frame using a standard personal computer. Typical optimization takes only a few minutes (approximately 40 iterations) to reach convergence (see Fig. 2i).
The second harmonic optimization ${ }^{19}$ generates a neardiffraction-limited focal spot as shown in Fig. 3a for the farfield laser intensity profile in vacuum. In Fig. 3a,b, we compare the transverse intensity distribution within the focal region over 
the length scale of the gas jet. The laser profile (Fig. 3b) that produces the best electron beam exhibits several low-intensity side lobes around the central peak, and has a peak intensity about half that of the optimized focus. The complex laser profiles appear to have a very dramatic effect on the structure of the plasma waves produced and consequently the electron beam profile. Figure $3 c$ shows the relative wavefront change recorded by a Shack-Hartmann wavefront sensor. Calculation using the reconstructed wavefront gives a Strehl ratio of about 0.5 , which is in agreement with the far-field intensity measurement. This small wavefront modification of the driver pulse can lead to a significant improvement in the electron beam properties through the relativistic nonlinear optics of the plasma. The relative position between the focal plane and the centre of the gas flow was controlled by moving the nozzle. Scanning the gas nozzle both before and after genetic algorithm confirms the optimal focal position does not change, excluding the possibility that the improvement may be due to optimizing the focal position.

Control of energy distribution. Furthermore, we extended the genetic algorithm optimization to control the electron energy distribution. Through control of the light propagation, the plasma wave amplitude will be affected and therefore also the strength of the accelerating gradient. Hence, we can expect to be able to modify the energy spectrum. A high-resolution energy analyser using a dipole magnet pair was used to obtain the electron energy spectrum as the electrons were dispersed in the horizontal plane in the magnetic field. A $150-\mu \mathrm{m}$ pinhole was placed $2.2 \mathrm{~cm}$ from the electron source to improve the energy resolution of the spectrometer. The schematic setup is shown in Fig. 4a. The energy resolution limited by the entrance pinhole and transverse

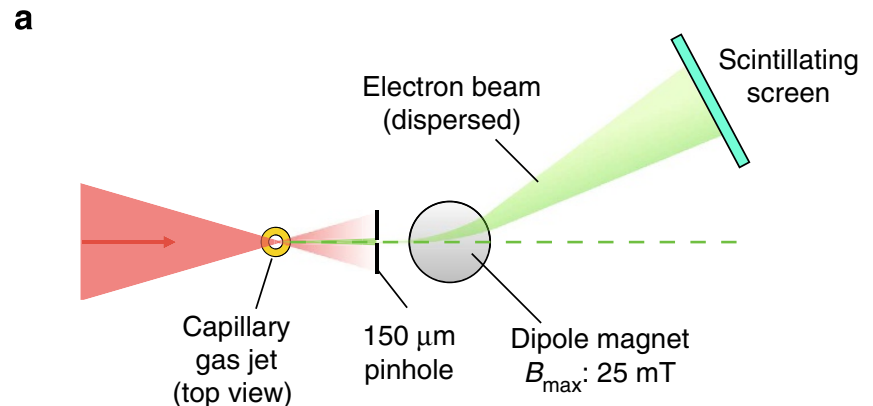

b
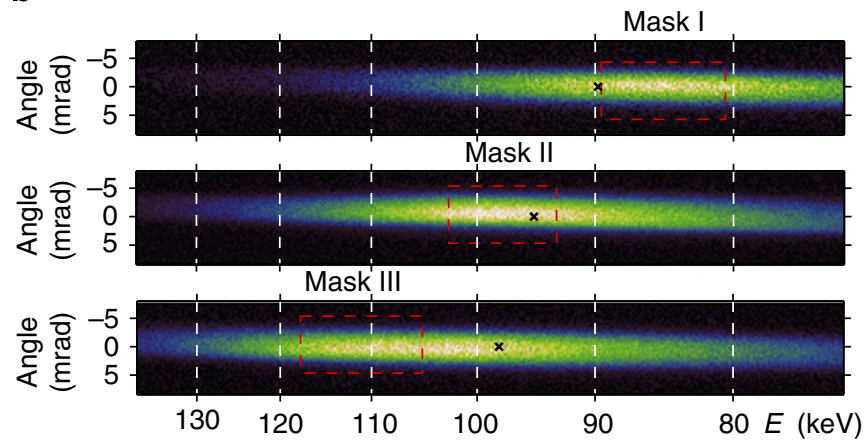

Figure 4 | Control of electron energy distribution. (a) Schematic setup for measuring electron energy distribution using dipole magnets. (b) Raw data showing the dispersed electron signal after genetic algorithm optimization using three different image masks. The location of the mask is indicated by the red rectangle and the black cross $(x)$ represents the final mean energy for each spectrum. 50 shots were integrated for each spectrum. emittance of the beam is estimated to be $2 \mathrm{keV}$ for the energy range of measurement.

Three rectangular masks are set in the low-, mid- and highenergy region on the dispersed data, namely masks I, II and III in Fig. 4b. We employed a fitness function (see Methods) to preferentially maximize the total counts inside the mask. Raw spectra from the genetic algorithm optimization are displayed in Fig. $4 \mathrm{~b}$, showing that the brightest part has shifted congruently. The resulting spectra have mean energies of 89,95 and $98 \mathrm{keV}$, respectively, for masks I, II and III, noting that they do not fall on the visual centroid of the image because the scintillator sensitivity is not included in the presentation of the raw data, however, it was taken into account for computing the mean energies. Our results show that manipulation of electron energy distribution using the deformable mirror is somewhat restricted. The final result after optimization does not reach the objective mask completely despite that the mean energies can be varied by up to $10 \%$. This result is somewhat unsurprising as the scope for controlling the electron spectrum is mostly limited by the physics of the interaction-while changing the transverse intensity profile can make big differences to the shape of the plasma electric field structure, changing the maximum field amplitude of the wakefield (and therefore peak energy of the accelerated electrons) will be limited.

Numerical simulations. Although the details of the initial conditions required for optimal beams are difficult to determine and are therefore found using the genetic algorithm, we can at least demonstrate how modifications to the phase front of the laser pulse can improve the beam properties with an example. To illustrate the underlying physics of the plasma wave dynamics determined by the conditions of the driving laser pulse, we performed two-dimensional (2D) particle-in-cell simulations using the OSIRIS framework ${ }^{26}$. Parameters similar to the experiment conditions were used, with a Gaussian plasma density profile to enable trapping of electrons in the density down ramp (see ref. 27 and Methods for details on the simulation).

It was previously shown in ref. 28 that the focusing fields of laser plasma accelerators can be controlled by tailoring the transverse intensity profile of the laser pulse using higher-order modes, where generalization to $3 \mathrm{D}$ was also discussed. Here, we simulated a laser pulse with a fundamental Gaussian mode $\left(\mathrm{TEM}_{00}\right)$ or a coherent superposition of a fundamental $\left(\mathrm{TEM}_{00}\right)$ and a second-order Hermite-Gaussian $\left(\mathrm{TEM}_{02}\right)$ mode (Fig. 5a). Although the plasma wave has a larger amplitude when it is driven by a single-mode laser pulse, the wake phase front evolves a backward curvature when electrons are trapped and accelerated (see top panel in Supplementary Movie 1 and Fig. 5b). Contrastingly, the evolution of the wakefield driven by the laser pulse with additional mode forms a flatter plasma phase front at the point of trapping (Fig. $5 \mathrm{~d}$ ).

In Fig. 5c,e, the momentum distribution of the forward accelerated electrons has a larger transverse spread for the singlemode laser pulse compared with the one with the addition of higher-order modes. This is a consequence of the different trapping conditions and accelerating fields from the coherent plasma wakefield structure, which is governed by the structure of the driving laser pulse. In a comparative test to show this effect is not simply due to a lower intensity, we repeated the simulation using a single fundamental Gaussian mode laser pulse with a larger focal spot with the same peak intensity as that with the superimposed modes. The wakefield evolution shows very similar response as Fig. 5b and does not develop a flatter phase front as seen in Fig. 5 d. The subsequently accelerated electrons have very similar divergence to that in Fig. $5 c$, eliminating the possibility 
a

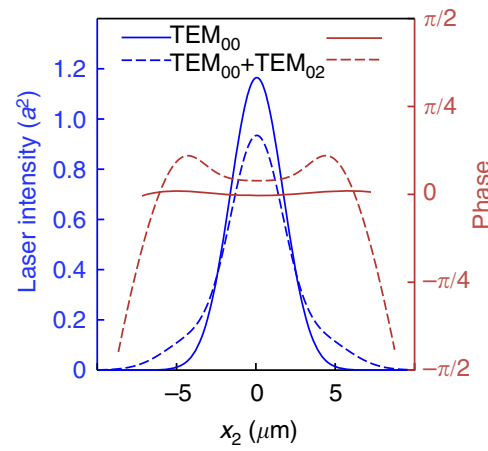

b

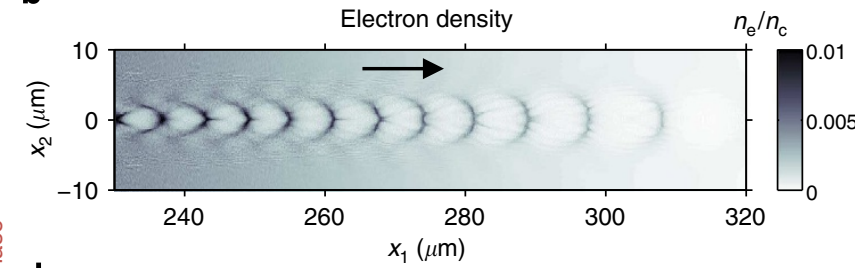

C
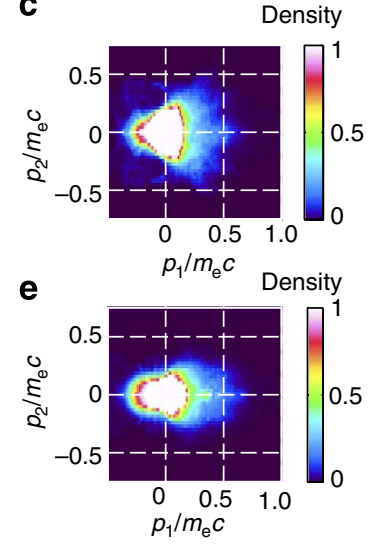

Figure 5 | Modelling the effect of laser phase front using coherently superimposed modes. (a) Transverse laser intensity profile and phase at the focal plane $\left(x_{1}=215 \mu \mathrm{m}\right)$ for a single Gaussian mode (solid curves) and a superimposed mode (dashed curves) obtained in the $2 \mathrm{D}$ simulations. Plotted are the laser pulses at vacuum focus. (b,d) Snapshots showing the different plasma wave structures around the time ( $t=1.28$ ps) and location of trapping, driven by a laser pulse of single mode (b) and superimposed mode (d). The laser pulse propagates to the right. The phase space $p_{2}-p_{1}$ distribution of the accelerated electrons shown for $(\mathbf{c})$ single mode and (e) superimposed mode before the electrons exit the simulation box $(t=2.25$ s).

that the improvement comes from either a high-intensity effect or a simple change in $f$-number. Note that we are not saying that this mixed-Hermite-Gaussian mode is the optimal pulse in the experiment; this is simply an illustration of how small changes in pulse shape can have significant effects on electron beam properties.

\section{Discussion}

When a particular wavefront of laser light interacts with plasma, it can affect the plasma wave structures and trapping conditions of the electrons in a complex way. For example, Raman forward scattering, envelope self-modulation, relativistic self-focusing and relativistic self-phase modulation ${ }^{29}$ and many other nonlinear interactions modify both the pulse envelope and phase as the pulse propagates, in a way that cannot be easily predicted and that subsequently dictates the formation of plasma waves. Moreover, under realistic experimental conditions, ionization dynamics before the laser pulse reaches the vacuum focus can also modify the phase of the driving pulse. Ideally, the light interacts in such a way as to generate large amplitude plasma waves with electric field structures that accelerate electrons with small divergence, high charge and so on. Because of the complicated interaction, it is difficult to determine a laser phase profile that will lead to such a plasma structure. However, such unforeseeable conditions were successfully revealed by using the evolutionary algorithm method, with the result that the electron charge can be increased and emitted in a very well collimated beam.

Here we have implemented coherent control of a nonlinear plasma wave and demonstrated an order of magnitude improvement in the electron beam parameters. The laser beam optimized to generate the best electron beam was not the one with the 'best' focal spot. Control and shaping of the electron energy distribution was observed to be less effective, but was still possible. The capability for wavefront control was also limited by the number of actuators and maximum deformation of the deformable mirror used in our experiments. In addition, this work was performed using adaptive optics, but it is clear that coherent control of plasma waves should be possible in a variety of configurations, for example, by using an acousto-optic modulator to control the temporal phase of the driving pulse. Recently developed techniques ${ }^{30,31}$ for single-shot diagnosis of plasma wave structures may provide an avenue for direct control of the plasma evolution.
The concept of coherent control for plasmas opens new possibilities for future laser-based accelerators. Although still at the stage of fundamental research, laser wakefield accelerators are showing significant promise. In principle, such improvements could be integrated into next-generation high-power laser projects, such as the International Coherent Amplification Network $(\text { ICAN })^{32}$, based on coherent combination of many independent fibres, taking advantage of both their high repetition rate and controllability. The stability and response of the wakefield to laser conditions, such as phase front errors, is not well understood, but is crucial for the success of laser wakefield acceleration as a source of relativistic electrons and secondary radiation. For example, the presence of an asymmetric laser pulse was shown to affect the betatron oscillations and properties of X-rays produced in laser wakefield accelerators ${ }^{33-36}$. Implementing the methods of this study should enable a significantly improved understanding and control of the wakefield acceleration process with regard to stability, dark current reduction and beam emittance.

\section{Methods}

Laser system. The Relativistic Lambda Cubed laser $\left(\lambda^{3}\right)$ produces $30 \mathrm{fs}$ pulses of $800 \mathrm{~nm}$ light at a repetition rate of $500 \mathrm{~Hz}$ with an ASE (Amplified-SpontaneousEmission) intensity contrast of $\sim 10^{8}$ around $1 \mathrm{~ns}$ before the main pulse. The system is seeded by a Femto-Laser Ti:sapphire oscillator, which generates $12 \mathrm{fs}$ pulses and has a companion carrier envelope phase locking system. An RF addressable acousto-optic filter called a Dazzler controls the spectral amplitude and phase of these pulses. Selected pulses from the Dazzler train are stretched to $220 \mathrm{ps}$ in a low-aberration stretcher and amplified to $7 \mathrm{~mJ}$ in a cryogenically cooled largemode regenerative amplifier (Regen). The energy dumped from the Regen cavity is 'cleaned' in a Pockels cell and used to seed a 3-pass amplifier as an upgrade from the laser system described in ref. 37 , which delivers up to $28 \mathrm{~mJ}$ pulses before compression. Following $71 \%$ efficient compression, $20 \mathrm{~mJ}$ pulses are trimmed to $18 \mathrm{~mJ}$ at the perimeter of a $47-\mathrm{mm}$ diameter, 37 -actuator deformable mirror. Throughout the system, pump light is provided by a variety of internally doubled Nd-doped yttrium aluminium garnet (YAG), yttrium lithium fluoride (YLF) and vanadate lasers. The output beam with its controllable wavefront is then delivered to one of five experimental areas for the production of X-rays, electron beams, ion beams, $\mathrm{THz}$ radiation, high-order harmonics or warm-dense matter.

Electron acceleration and detection. The focused laser pulse drives plasma waves by interacting with an argon gas jet flowing continuously from a $100-\mu \mathrm{m}$ inner diameter fused silica capillary. Typically, the laser axis is $300 \mu \mathrm{m}$ above the orifice of the tubing. The laser pulses were focused by an $f / 2$ off-axis parabolic mirror to a spot size of $2.5 \mu \mathrm{m}$ FWHM with a maximum of $10 \mathrm{~mJ}$ energy on target. The plasma electron density is measured to be in the range $(0.5-2) \times 10^{19} \mathrm{~cm}^{-3}$ using 
transverse interferometry. Electrons are accelerated in the density down ramp with a final energy in the $100-\mathrm{keV}$ range ${ }^{27}$ and detected by a high-resolution scintillating screen (J6677 FOS by Hamamatsu), which is placed about $35 \mathrm{~cm}$ downstream from the source and imaged with a lens coupled 12-bit CCD camera for a $4 \times 4 \mathrm{~cm}^{2}$ effective area. The scintillator sensitivity was calibrated using an electron microscope for the energy range in the spectrum measurement. Electron beam charge was estimated using the calibrated scintillator response, manufacturerprovided information for the CCD camera (gain, quantum efficiency and so on) and the measured effective numerical aperture of the imaging system.

Focal characterization and wavefront measurement. The amplified laser beam was attenuated by using a half-wave plate and the polarization-dependent properties of the compressor grating of the laser system. $\varnothing 25 \mathrm{~mm}$ neutral density filters (Thorlabs, Inc.) were inserted in the exit beam after the compressor and before a telescope beam expander. The laser focus was imaged by a $\times 60$ microscope objective lens (Newport Corporation, M-60X) onto a 8-bit CCD camera for focal characterization (cf setup A in Fig. 1). A Shack-Hartmann wavefront sensor (Flexible Optical BV) was used to determine the relative wavefront change between different deformable mirror configurations. The sensor, which consists of a $30 \times 30$ microlens array having a focal length of $3.5 \mathrm{~mm}$ and $150 \mu \mathrm{m}$ pitch, was directly placed in the path of the converging beam after the focusing parabolic mirror. Reconstruction of the wavefront was performed using the FrontSurfer analysis software (Flexible Optical BV), typically with $\approx 450$ measured local wavefront slopes and root-mean-square (RMS) error on the order of $0.05 \lambda$. Rotating the neutral density filters and the half-wave plate did not change the focal spot or the wavefront measurement significantly, insuring the wavefront distortion introduced by attenuation was negligible.

Deformable mirror and genetic algorithm. The deformable mirror (AOA Xinetics) has a $47-\mathrm{mm}$ clear aperture of a continuous face sheet with 37 piezoelectric actuators arranged on a square grid spaced $7 \mathrm{~mm}$ apart. The maximum stroke used in this experiment is about $2 \mu \mathrm{m}$.

The mirror shape is controlled by a genetic algorithm, which is a method mimicking the process of natural selection and routinely used to generate optimal solutions in complex systems with a large number of variables. The genetic representation in our experiments comprises a set of 37 independent voltage values for the deformable mirror actuator array. A fitness function is designed to produce a single FOM to evaluate how close the solution is to the goal. follows:

In the electron beam profile optimization experiment, FOM is computed as

$$
\mathrm{FOM}=\sum_{\substack{(i, j) \\ \mathbf{r}_{\mathbf{i j}} \neq \mathbf{r}_{0}}} \frac{I_{i j}}{\left|\mathbf{r}_{\mathbf{i j}}-\mathbf{r}_{0}\right|^{n}}
$$

where $I_{i j}$ is the pixel intensity for every pixel $(i, j)$ in the whole image and $\mathbf{r}_{0}$ is a coordinate point in the image used as an optimization target. The power factor $n>0$ gives higher weighting to those pixels closer to the target (inverse distance weighting).

In the experiment to control the energy spectrum, FOM is calculated using the following formula given a pre-defined image mask,

$$
\text { FOM }=\left(1-\frac{\text { mean intensity outside mask }}{\text { mean intensity of whole image }}\right) \times \text { mean intensity inside mask }
$$

The mean intensity is the sum of the pixel counts divided by the number of pixels for a defined region. A rectangular mask was used in the experiment as specified by the region enclosed by the red dashed lines in Fig. $4 \mathrm{~b}$.

Numerical simulations. The $2 \mathrm{D}$ particle-in-cell simulations were performed in a stationary box of the dimensions $573 \times 102 \mu \mathrm{m}$ with $10,000 \times 600$ cells and $4 \times 4$ particles-per-cell. A Gaussian plasma density profile was used in the propagation dimension $\left(x_{1}\right)$, peaked at $x_{1}=200 \mu \mathrm{m}$ with a FWHM of $120 \mu \mathrm{m}$ and a maximum electron density of $0.005 n_{c}$, where $n_{c}$ is the plasma critical density. The laser pulse was initialized at the left edge of the simulation window and focused at $215 \mu \mathrm{m}$ in the density down ramp. In $2 \mathrm{D}$ geometry, the transverse intensity profile of the laser pulse for fundamental Gaussian mode $\left(\mathrm{TEM}_{00}\right)$ has the form $a_{0}^{2} \exp \left(-2 x_{2}^{2} / w_{0}^{2}\right)$, and the second-order Hermite-Gaussian mode $a_{2}^{2} \exp \left(-2 x_{2}^{2} / w_{2}^{2}\right)\left(8 x_{2}^{2} / w_{2}^{2}-2\right)^{2}$, where $a_{0,2}$ is the normalized vector potential and $w_{0,2}$ is the beam waist parameter. The two modes are coherently superimposed in the same plane of polarization. Here we used even-order Hermite-Gaussian mode $\left(\mathrm{TEM}_{02}\right)$ for its symmetric property. A phase difference of $\pi / 8$ was applied at the beam waist between the two modes to simulate variations in the optical phase front condition. The beam waist was positioned to account for focal shift as a result of coherent superposition of two modes such that the location of the maximum on-axis laser intensity was the same (at $x_{1}=215 \mu \mathrm{m}$ ) for all simulation runs. The simulation parameters are $a_{0}=1.08$ and $w_{0}=3.31 \mu \mathrm{m}$ for the Gaussian mode alone, or $a_{0}=1.0, a_{2}=0.15$ and $w_{0}=w_{2}=3.31 \mu \mathrm{m}$ for the superimposed mode.

\section{References}

1. Warren, W. S., Rabitz, H. \& Dahleh, M. Coherent control of quantum dynamics: the dream is alive. Science 259, 1581-1589 (1993).

2. Wineland, D. et al. Experimental issues in coherent quantum-state manipulation of trapped atomic ions. J. Res. Natl Inst. Stand. Technol. 103, 259 (1998).

3. Assion, A. et al. Control of chemical reactions by feedback-optimized phase-shaped femtosecond laser pulses. Science 282, 919-922 (1998).

4. Nakamura, Y., Pashkin, Y. \& Tsai, J. Coherent control of macroscopic quantum states in a single-cooper-pair box. Nature 398, 786-788 (1999).

5. Reithmaier, J. et al. Strong coupling in a single quantum dot-semiconductor microcavity system. Nature 432, 197-200 (2004).

6. Press, D., Ladd, T. D., Zhang, B. \& Yamamoto, Y. Complete quantum control of a single quantum dot spin using ultrafast optical pulses. Nature 456, 218-221 (2008).

7. Xie, X., Dai, J. \& Zhang, X.-C. Coherent control of THz wave generation in ambient air. Phys. Rev. Lett. 96, 075005 (2006).

8. Faure, J. et al. Observation of laser-pulse shortening in nonlinear plasma waves Phys. Rev. Lett. 95, 205003 (2005).

9. Schreiber, J. et al. Complete temporal characterization of asymmetric pulse compression in a laser wakefield. Phys. Rev. Lett. 105, 235003 (2010).

10. Malkin, V. M., Shvets, G. \& Fisch, N. J. Fast compression of laser beams to highly overcritical powers. Phys. Rev. Lett. 82, 4448-4451 (1999).

11. Trines, R. M. G. M. et al. Simulations of efficient Raman amplification into the multipetawatt regime. Nat. Phys. 7, 87-92 (2011).

12. Malkin, V. M. \& Fisch, N. J. Collective deceleration of relativistic electrons precisely in the core of an inertial-fusion target. Phys. Rev. Lett. 89, 125004 (2002).

13. Willingale, L. et al. High-power, kilojoule class laser channeling in millimeterscale underdense plasma. Phys. Rev. Lett. 106, 105002 (2011).

14. Tajima, T. \& Dawson, J. M. Laser electron accelerator. Phys. Rev. Lett. 43, 267-270 (1979).

15. Mangles, S. P. D. et al. Monoenergetic beams of relativistic electrons from intense laser-plasma interactions. Nature 431, 535-538 (2004).

16. Geddes, C. et al. High-quality electron beams from a laser wakefield accelerator using plasma-channel guiding. Nature 431, 538-541 (2004).

17. Faure, J. et al. A laser-plasma accelerator producing monoenergetic electron beams. Nature 431, 541-544 (2004).

18. Bahk, S.-W. et al. Generation and characterization of the highest laser intensities $\left(10^{22} \mathrm{~W} / \mathrm{cm}^{2}\right)$. Opt. Lett. 29, 2837-2839 (2004).

19. Albert, O., Wang, H., Liu, D., Chang, Z. \& Mourou, G. Generation of relativistic intensity pulses at a kilohertz repetition rate. Opt. Lett. 25, 1125-1127 (2000)

20. Planchon, T. A. et al. Adaptive correction of a tightly focused, high-intensity laser beam by use of a third-harmonic signal generated at an interface. Opt. Lett. 31, 2214-2216 (2006).

21. Zeng, Z., Ling, N. \& Jiang, W. The investigation of controlling laser focal profile by deformable mirror and wave-front sensor. J. Mod. Opt. 46, 341-348 (1999).

22. El-Agmy, R., Bulte, H., Greenaway, A. H. \& Reid, D. Adaptive beam profile control using a simulated annealing algorithm. Opt. Express 13, 6085-6091 (2005).

23. Campbell, S., Triphan, S. M. F., El-Agmy, R., Greenaway, A. H. \& Reid, D. T Direct optimization of femtosecond laser ablation using adaptive wavefront shaping. J. Opt. A Pure Appl. Opt. 9, 1100 (2007).

24. Bartels, R. et al. Shaped-pulse optimization of coherent emission of highharmonic soft X-rays. Nature 406, 164-166 (2000).

25. Yoshitomi, D. et al. Phase-matched enhancements of high-harmonic soft x-rays by adaptive wave-front control with a genetic algorithm. Appl. Phys. B 78, 275-280 (2004).

26. Fonseca, R. A. et al. OSIRIS: a three-dimensional, fully relativistic particle in cell code for modeling plasma based accelerators. Lect. Notes Comp. Sci. 2331, 342-351 (2002).

27. He, Z.-H. et al. High repetition-rate wakefield electron source generated by fewmillijoule, $30 \mathrm{fs}$ laser pulses on a density downramp. N. J. Phys. 15, 053016 (2013).

28. Cormier-Michel, E. et al. Control of focusing fields in laser-plasma accelerators using higher-order modes. Phys. Rev. ST Accel. Beams 14, 031303 (2011).

29. Mori, W. The physics of the nonlinear optics of plasmas at relativistic intensities for short-pulse lasers. IEEE J. Quantum Electronics 33, 1942-1953 (1997).

30. Li, Z. et al. Single-shot visualization of evolving laser wakefields using an all-optical streak camera. Phys. Rev. Lett. 113, 085001 (2014).

31. Sävert, A. et al.Direct imaging of the dynamics of a laser-plasma accelerator operating in the bubble-regime. Preprints at http://arxiv.org/abs/1402.3052 (2014).

32. Mourou, G., Brocklesby, B., Tajima, T. \& Limpert, J. The future is fibre accelerators. Nat. Photon 7, 258-261 (2013).

33. Glinec, Y. et al. Direct observation of betatron oscillations in a laser-plasma electron accelerator. Europhys. Lett. 81, 64001 (2008).

34. Mangles, S. P. D. et al. Controlling the spectrum of $\mathrm{x}$-rays generated in a laser-plasma accelerator by tailoring the laser wavefront. Appl. Phys. Lett. 95, 181106 (2009). 
35. Popp, A. et al. All-optical steering of laser-wakefield-accelerated electron beams. Phys. Rev. Lett. 105, 215001 (2010).

36. Schnell, M. et al. Optical control of hard X-ray polarization by electron injection in a laser wakefield accelerator. Nat. Commun. 4, 2421 (2013).

37. Hou, B., Easter, J., Mordovanakis, A., Krushelnick, K. \& Nees, J. A.

Vacuum-free X-ray source based on ultrashort laser irradiation of solids. Opt. Express 16, 17695-17705 (2008).

\section{Acknowledgements}

This work was supported by MCubed at the University of Michigan, the AFOSR Young Investigator Program (Grant No. FA9550-12-1-0310), DARPA (Contract No. N66001-

11-1-4208), the NSF (Grant No. 0935197) and NSF CAREER (Grant No 1054164). We acknowledge the OSIRIS Consortium, consisting of University of California, Los Angeles (UCLA) and Instituto Superior Técnico (IST) (Lisbon, Portugal), for the use of the OSIRIS 2.0 framework. This research was supported in part through computational resources and services provided by Advanced Research Computing at the University of Michigan, Ann Arbor.

\section{Author contributions}

Z.-H.H., B.H., J.A.N. designed and carried out the experiment. Z.-H.H. performed all data analysis and the simulations. V.L. and B.H. developed the LabView program. K.K. and A.G.R.T. directed and guided the project. Z.-H.H. and A.G.R.T. wrote the paper. All authors discussed the results and contributed to the manuscript.

\section{Additional information}

Supplementary Information accompanies this paper at http://www.nature.com/ naturecommunications

Competing financial interests: The authors declare no competing financial interests.

Reprints and permission information is available online at http://npg.nature.com/ reprintsandpermissions/

How to cite this article: He, Z.-H. et al. Coherent control of plasma dynamics. Nat Commun. 6:7156 doi: 10.1038/ncomms8156 (2015). 